\title{
Universal behavior of a trapped Fermi superfluid in the BCS-unitarity crossover
}

\author{
S. K. Adhikari \\ Instituto de Física Teórica, UNESP-São Paulo State University, 01.405-900 São Paulo, São Paulo, Brazil
}

(Received 5 October 2008; published 9 February 2009)

\begin{abstract}
From an extensive calculation of static properties of a trapped Fermi superfluid at zero temperature using a density-functional formulation, we demonstrate a universal behavior of its observables, such as energy, chemical potential, radius, etc., over the crossover from the BCS limit to unitarity leading to scaling over many orders of magnitude in fermion number. This scaling allows us to predict the static properties of the system, with a large number $\left(\sim 10^{5}\right)$ of fermions, over the crossover with an error of $1 \%-2 \%$, from the knowledge of those for a small number $(\sim 10)$ of fermions.
\end{abstract}

DOI: 10.1103/PhysRevA.79.023611

PACS number(s): 67.85.Bc, 03.75.Ss, 05.30.Fk, 71.10.Ay

\section{INTRODUCTION}

The Pauli principle leads to an effective repulsion in identical fermions which could dominate the physical interaction and control the dynamics. The dominance of Pauli repulsion is responsible for the stability of our hadronic universe. When this happens the system exhibits universal behavior practically independent of or weakly dependent on the physical interaction. One classic example [1] of this is found in the $s$-wave quartet nucleon-deuteron system with three spinparallel nucleons controlled by Pauli repulsion. The calculated scattering length for different nucleon-nucleon interactions is essentially $6.3 \mathrm{fm}$, whereas the doublet $s$-wave scattering length (not controlled by Pauli repulsion) for the same interactions varies from $-2 \mathrm{fm}$ to $3 \mathrm{fm}[1,2]$. This universality is prominent in the limit of zero Fermi-Fermi interaction in the BCS theory of superfluid fermions leading to universal properties of low-temperature superconductors [3], of cold neutron matter and neutron star [4,5], and of a trapped Fermi superfluid [6] at zero temperature. This universality also manifests [7-10] in a trapped Fermi superfluid at unitarity as the Fermi-Fermi scattering length $a$ goes to infinity $(a \rightarrow-\infty)$. In this limit, though the physical interaction is nonzero, the only interaction scale (scattering length) disappears and the system acquires universal behavior. This interaction scale is also absent in the BCS limit $(a=-0)$ with universal properties. Similar universality is found in other fermionic systems with large $|a|[11]$.

The crossover from weak-coupling BCS limit to unitarity [12] has been a very active area of research $[5,7,10,13]$ after the experimental realization $[14,15]$ of this crossover in a trapped dilute Fermi superfluid near a Feshbach resonance. Using a complete numerical simulation of a densityfunctional (DF) formulation [16], we show that the deviation from universality of a trapped dilute Fermi superfluid over the crossover is orderly and the system continues to exhibit nearly universal behavior possessing useful scaling relations over many orders of magnitude involving energy, chemical potential, radius, and number of Fermi atoms $(N)$. In this crossover region the Pauli repulsion dominates over the physical interaction leading to the universal behavior.

In Sec. II we present the density-functional formulation that we use in this study. In Sec. III we present the numerical results and establish the universal behavior of a trapped
Fermi superfluid in the BCS-unitarity crossover. Finally, in Sec. IV we give some concluding remarks.

\section{DENSITY-FUNCTIONAL FORMULATION}

To study the universality, we use a Galilei-invariant DF formulation for the crossover of a trapped two-component Fermi superfluid [17], equivalent to a hydrodynamical model with the correct phase-velocity relation [6] $\mathbf{v}=\hbar \nabla \theta /(2 m)$, where $\mathbf{v}$ is the superfluid velocity, $m$ the Fermi mass, and $\theta$ the phase of the order parameter $\Psi(\mathbf{r})$ at position $\mathbf{r}$, which satisfies [Eq. (35) of [17], but with a distinct $g(x)$ consistent with the known small- $x$ behavior of energy of a uniform Fermi gas]

$$
\begin{gathered}
\left(-\frac{\hbar^{2}}{8 m} \nabla^{2}+U+\mu(n, a)\right) \Psi(\mathbf{r})=\mu_{0} \Psi(\mathbf{r}), \\
\mu(n, a)=\frac{\hbar^{2}}{2 m}\left(3 \pi^{2} n\right)^{2 / 3} g\left(n^{1 / 3} a\right), \\
\mu_{0}=\int d \mathbf{r}\left(\frac{\hbar^{2}}{8 m}|\nabla \Psi|^{2}+U \Psi^{2}+\mu(n, a) \Psi^{2}\right),
\end{gathered}
$$

where $\int \Psi^{2}(\mathbf{r}) d \mathbf{r}=N$, and

$$
g(x)=1+\frac{\left(\chi_{1} x-\chi_{2} x^{2}\right)}{\left(1-\beta_{1} x+\beta_{2} x^{2}\right)},
$$

with $\chi_{1}=4 \pi /\left(3 \pi^{2}\right)^{2 / 3}, \quad \chi_{2}=300, \quad \beta_{1}=40, \quad \beta_{2}=\chi_{2} /(1-\zeta)$, $\mu(n, a)$ the bulk chemical potential, $\mu_{0}$ the chemical potential for the trapped system, $n=\Psi^{2}$ the density of atoms, and $U=m \omega^{2} r^{2} / 2$ the harmonic trap of frequency $\omega$. [Different parametrizations of $g(x)$ were used in Ref. [18].] Here we take $\zeta=0.44$ consistent with Monte Carlo calculations $[5,13]$ and experiments [19] of a uniform Fermi superfluid at unitarity. The parameters $\chi_{1}, \chi_{2}, \beta_{1}$, and $\beta_{2}$ are chosen so that the model (i) agrees with the fixed-node Monte Carlo (FNMC) [7] and Green-function Monte Carlo (GFMC) [20] results for the energy of a trapped superfluid at unitarity (for $N<30$ ) and over the crossover [7,17], (ii) provides a smooth interpolation between the energies of a superfluid at the BCS and unitarity limits $[17,21]$, and (iii) satisfies the known BCS limit [22] of the bulk chemical potential $\mu(n, a)$ [two lowestorder terms of Eq. (1) of [23]]. 


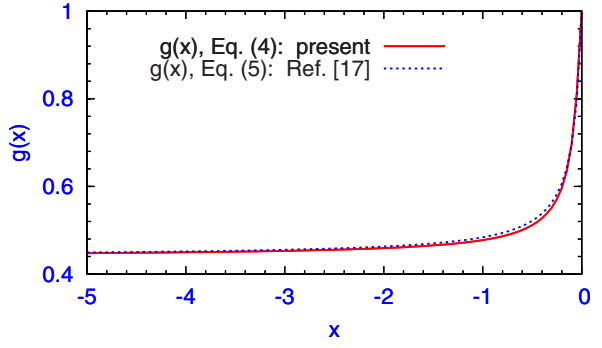

FIG. 1. (Color online) The function $g(x)$ vs $x$ as defined by Eqs. (4) and (5).

In Ref. [17] we used the following simple expression for the function $g(x)$ in place of that given by Eq. (4):

$$
g(x)=1+\frac{\chi x}{1-\beta x},
$$

with $\chi=20 \pi /\left(3 \pi^{2}\right)^{2 / 3}$, and $\beta=\chi /(1-\zeta)$. This choice does not satisfy the known weak interaction BCS $\operatorname{limit} \lim _{x \rightarrow 0} g(x)$ $\rightarrow 1+4 \pi x /\left(3 \pi^{2}\right)^{2 / 3}[17,18,23]$. Equation (5) was used in Ref. [17] as a simple model to satisfy the BCS-unitarity crossover Monte Carlo results [21] for energies of trapped fermions. The bulk energy (and chemical potential) of a Fermi gas in the weak-couping BCS limit can be written as an expansion in the parameter $x=n^{1 / 3} a$ as discussed in Ref. [23]. Lee and Yang [22] calculated the coefficients of the series in a specific model of interacting fermions as quoted in Eq. (1) of [23]. These coefficients can be related to the coefficients in an expansion of $g(x)$ in the small $x$ limit. The $x$-independent constant in $g(x)$ and the coefficient of the $x$ term $\chi$ $=4 \pi /\left(3 \pi^{2}\right)^{2 / 3}$ in this series are reasonably model independent. However, coefficients of $x^{2}$ will be dependent on the interaction model. In Ref. [17] we changed the coefficient of the $x$ term from that proposed in [23], to simulate the effect of the (unknown) higher-order terms in the $x$ expansion of $g(x)$. The coefficient of the $x$ term can be kept at the LeeYang value $4 \pi /\left(3 \pi^{2}\right)^{2 / 3}$ provided we include a $x^{2}$ term in the expansion of $g(x)$ and we have done this in Eq. (4). However, the coefficient of the present $x^{2}$ term does not and should not agree with that of the known expansion quoted in Eq. (1) of [23]. This is because this coefficient now simulates the contribution of higher-order terms. Although the analytic expression $g(x)$ is modified here from that given by Eq. (5) to (4), from a numerical (calculational) point of view the change is negligible as we can see from Fig. 1, where we plot the two parametrizations of the function $g(x)$ given by Eqs. (4) and (5). In Ref. [17], we used the function $g(x)$ of Eq. (5) to reproduce some Monte Carlo results [21] for energy of a trapped-fermion system over the BCS to unitarity crossover. We have now performed the same calculations with the function $g(x)$ of Eq. (4) - illustrated below in Fig. 4(b) - and we verified that the results for energy remain practically unchanged.

The gradient term in Eq. (1) provides a correction to the local density approximation (LDA) [24] obtained by setting the gradient term to zero. LDA is a good approximation for a large $N$, when the bulk chemical potential $\mu(n, a)$, a positive term responsible for Pauli repulsion in the system even for attractive (negative) $a$, is very large. The gradient term is consistent with the hydrodynamic flow of paired fermions of mass $2 m[6,17]$. To study the scaling of the solution of Eq. (1) we note that in the BCS and unitarity limits the bulk chemical potential $\mu(n, a)$ has, respectively, the following simple forms $[5,6,13]$ : $\hbar^{2}\left(3 \pi^{2} n\right)^{2 / 3} /(2 m)$ and $\zeta \hbar^{2}\left(3 \pi^{2} n\right)^{2 / 3} /(2 m)$, which shows the scaling $\mu(n, a) \sim n^{2 / 3}$ in both limits. In these limits the energy functional is given by [6]

$$
\begin{aligned}
E & =\int d \mathbf{r}\left(\frac{\hbar^{2}}{8 m}|\nabla \Psi|^{2}+U \Psi^{2}+\frac{3 \hbar^{2} \xi}{20 m}\left(3 \pi^{2}\right)^{2 / 3} \Psi^{10 / 3}\right), \\
& \equiv\left\langle E_{\boldsymbol{\nabla}}\right\rangle+\left\langle E_{\mathrm{pot}}\right\rangle+\left\langle E_{\mathrm{Fermi}}\right\rangle,
\end{aligned}
$$

where $\xi=1$ in the BCS limit and $\xi=\zeta=0.44$ at unitarity $[5,13]$, and $\left\langle E_{\boldsymbol{\nabla}}\right\rangle,\left\langle E_{\text {pot }}\right\rangle$, and $\left\langle E_{\mathrm{Fermi}}\right\rangle$ are, respectively, the expectation values of the three terms in Eq. (6). This "analytic" dependence of $E$ on $\Psi$ leads to a simple virial theorem, postulated and studied experimentally in [25], connecting $\left\langle E_{\boldsymbol{\nabla}}\right\rangle,\left\langle E_{\mathrm{pot}}\right\rangle$, and $\left\langle E_{\mathrm{Fermi}}\right\rangle$ at the BCS and unitarity limits. For the exact $\Psi$, energy $E$ is a minimum. To derive the virial theorem, we take the Cartesian system $\mathbf{r} \equiv(x, y, z)$, and the norm-preserving scaling transformation $\Psi(x, y, z)$ $\rightarrow \sqrt{\lambda} \psi(\lambda x, y, z)$. The condition of minimum energy is $|d E / d \lambda|_{\lambda=1}=0$. The $\lambda$-dependent part of energy now becomes $E_{\lambda}=\lambda^{2}\left\langle E_{\nabla}^{x}\right\rangle+\lambda^{-2}\left\langle E_{\text {pot }}^{x}\right\rangle+\lambda^{2 / 3}\left\langle E_{\text {Fermi }}\right\rangle$, where the suffix $x$ denotes $x$ component. The minimization condition yields $\left\langle E_{\text {pot }}^{x}\right\rangle=\left\langle E_{\nabla}^{x}\right\rangle+\left\langle E_{\mathrm{Fermi}}\right\rangle / 3$. By summing over three components, we have the virial theorem $\left\langle E_{\mathrm{pot}}\right\rangle=\left\langle E_{\boldsymbol{\nabla}}\right\rangle+\left\langle E_{\mathrm{Fermi}}\right\rangle$, or equivalently, $E=2\left\langle E_{\text {pot }}\right\rangle$ at the BCS and unitarity limits. The deviation in percentage from the virial theorem in the crossover can be estimated by the percentage defect function $\eta$ $=100\left(E-2\left\langle E_{\text {pot }}\right\rangle\right) / E$.

In the LDA one has the following analytic solutions for the energy, chemical potential, and mean-square radius of the trapped system [24]: $\quad E /(\hbar \omega)=(3 N)^{4 / 3} \sqrt{\xi} / 4, \quad \mu_{0} /(\hbar \omega)$ $=(3 N)^{4 / 3} \sqrt{\xi / 3},\left\langle r^{2}\right\rangle /(\hbar / m \omega)=(3)^{4 / 3} N^{1 / 3} \sqrt{\xi} / 4$. The absence of the parameter $a$ in $\mu(n, a)$ of Eq. (2) in the BCS and unitarity limits leads to the following properties: (i) the scaling $\mu(n, a) \sim n^{2 / 3}$ for the uniform Fermi superfluid, (ii) scaling $E /(\hbar \omega), \mu_{0} /(\hbar \omega) \sim N^{4 / 3}$, and (iii) the virial theorem, $E$ $=2\left\langle E_{\mathrm{pot}}\right\rangle$ for the trapped Fermi superfluid. These universal properties lead to predictability of the Fermi superfluid in the BCS and unitarity limits, e.g., predicting the energy for a large number of fermions from the knowledge of that for a small number of fermions obtained by accurate "exact" calculation. This is important as exact calculations for large systems are very difficult, if not impossible.

To extend the above predictability over the crossover, where analytical (LDA) results are not available, we study the deviation from the universal properties of a Fermi superfluid in the crossover region. We find that the deviation is orderly, which allows us to restore a universal and predictable behavior of the trapped Fermi superfluid, so that one can predict the properties of a large system from a knowledge of those for a small system. 
TABLE I. Dimensionless energies $E /\left[\hbar \omega(3 N)^{4 / 3}\right]$ of a trapped Fermi superfluid along the crossover. The last two columns, $\{4\}$ and $\{10\}$, give the predicted energies for $N=10^{5}$ employing scaling (8) using the energies for $N^{\prime}=4$ and 10 , respectively.

\begin{tabular}{lcccccc}
\hline \hline$a \backslash N$ & 10 & $10^{2}$ & $10^{4}$ & $10^{5}$ & $10^{5}\{4\}$ & $10^{5}\{10\}$ \\
\hline-0.001 & 0.2656 & 0.2539 & 0.2501 & 0.2499 & 0.2428 & 0.2470 \\
-0.01 & 0.2652 & 0.2531 & 0.2478 & 0.2459 & 0.2393 & 0.2434 \\
-0.1 & 0.2550 & 0.2364 & 0.2146 & 0.2046 & 0.2027 & 0.2049 \\
-1 & 0.2079 & 0.1869 & 0.1740 & 0.1714 & 0.1731 & 0.1740 \\
-10 & 0.1897 & 0.1731 & 0.1670 & 0.1665 & 0.1686 & 0.1693 \\
-100 & 0.1875 & 0.1714 & 0.1662 & 0.1659 & 0.1682 & 0.1688 \\
\hline \hline
\end{tabular}

\section{NUMERICAL RESULTS}

We solve Eq. (1) by transforming it to time-dependent form by replacing $\mu_{0}$ by a time derivative. We express energy variables in units of $\hbar \omega$, length in $\sqrt{\hbar /(m \omega)}$, and time in $\omega^{-1}$. The resultant equation is then discretized by the semiimplicit Crank-Nicholson algorithm using a typical space step 0.04 and time step 0.001 and then solved by imaginary time propagation. The chemical potential $\mu_{0}$ is then calculated via Eq. (3), energy in the BCS and unitarity limits via Eq. (6), and the energy in the crossover region via a numerically constructed energy functional. In Table I we display energy per particle in units of $E_{F}=(3 N)^{1 / 3} \hbar \omega$ (the Fermi energy of an ideal Fermi gas at the trap center) or $E /\left[\hbar \omega(3 N)^{4 / 3}\right]$ along the crossover for different $N$ and $a$. In Table II we report the respective chemical potentials $\mu_{0} /\left[\hbar \omega(3 N)^{4 / 3}\right]$.

To understand the universal nature of $E$ and $\mu_{0}$ of Tables I and II for different $N$, we study the nonlinear input $\mu(n, a)$ to Eq. (1). In Fig. 2(a) we plot $4 \pi^{2} n \mu(n, a) /\left(h^{2} n^{2 / 3}\right)$ vs $|a| n^{1 / 3}$ for $a<0$. In the $\operatorname{BCS}\left(|a| n^{1 / 3} \rightarrow 0\right)$ and unitarity $\left(|a| n^{1 / 3} \rightarrow \infty\right)$ limits one has the perfect scaling, $\mu(n, a)$ $\sim n^{2 / 3}$, with deviation from this behavior in the crossover region. Next, to study the behavior of $E$ and $\mu_{0}$ of the trapped Fermi superfluid, we plot in Fig. 2(b) the numerically calculated $E / N^{4 / 3}$ and $\mu_{0} / N^{4 / 3}$ vs $N$ for $a=-0,-0.5$, and $-\infty$ and the FNMC [7,9] and GFMC [20] results. Perfect scalings, $\mu_{0}, E \sim N^{4 / 3}$, are observed for large $N$ in the BCS and unitarity limits, with deviation in the crossover region and for small $N$. A careful analysis of the $\mu_{0}, E$ data of Fig. 2(b) for small $N$ reveals in the crossover region the average scalings $\left(E / N^{2 / 3}-0.37\right) \sim N^{2 / 3}$ and $\left(\mu_{0} / N^{2 / 3}-0.27\right) \sim N^{2 / 3}$. If these scalings were really perfect then plots of $\left(E / N^{2 / 3}\right.$
$-0.37) / N^{2 / 3}$ or $\left(\mu_{0} / N^{2 / 3}-0.27\right) / N^{2 / 3}$ vs $|a|$ for a fixed $N$ would lead to universal curves independent of $N$.

To quantify the deviation of the Fermi superfluid in the crossover region from universality, we study the deviation of our result for the trapped Fermi superfluid from the virial theorem, $E=2\left\langle E_{\mathrm{pot}}\right\rangle$. For this purpose we plot the function $\eta=100\left(E-2\left\langle E_{\text {pot }}\right\rangle\right) / E$ vs $|a|$ for different $N$ in Fig. 3(a). The shift of the curves with $N$ clearly shows that the results are dependent on $a$ and $N$. We find that the maximum deviation from the virial theorem in the crossover region is quite small and is about 4\%. A careful examination of Fig. 3(a) shows that this dependence on $|a|$ is linear in logarithmic scale and can be included by plotting $\eta$ vs $|\alpha|, \alpha=N^{1 / 6} a$, as can be seen from Fig. 3(b). In Fig. 3(b) we find that all curves for $\eta$ have collapsed essentially on a single curve. However, the curve for $N=10$ is a bit different from others, which confirms that there is a deviation from universality for smaller $N$. This $|\alpha|=\left|N^{1 / 6} a\right|$ dependence of properties of a trapped Fermi superfluid is quite universal as we see in the following.

Now we study the universality in $E$ and $\mu_{0}$ of the trapped Fermi superfluid obtained from a solution of Eq. (1). We plot the function $\delta \equiv\left[E(N, a) / N^{2 / 3}-0.37\right] / N^{2 / 3}$ vs $|a|$ for different $N$ in Fig. 4(a). [We recall that the universal nature of the function $\delta$ was obtained from an analysis of results in Fig. 2(b)]. We find that as in Fig. 3(a) the results for different $N$ are distinct. If the system were really dominated by universality, a plot of the scaled quantities $\kappa \equiv\left[E(N, \alpha) / N^{2 / 3}\right.$ $-0.37] / N^{2 / 3}$ vs $|\alpha|$ would lead to universal curves. This is indeed found in Fig. 4(b), where we also included the FNMC results [7] for small $N=4,8$. For small $N$ there is some deviation from universality which disappears for $N>10$. We note that in Fig. 4(b) the $N=30$ and in Fig. 2(b) the large- $N$ FNMC and GFMC data lie on the universal curves. These

TABLE II. Same as Table I for the chemical potential $\mu_{0}$.

\begin{tabular}{lcccccc}
\hline \hline$a \backslash N$ & 10 & $10^{2}$ & $10^{4}$ & $10^{5}$ & $10^{5}\{4\}$ & $10^{5}\{10\}$ \\
\hline-0.001 & 0.3449 & 0.3361 & 0.3333 & 0.3331 & 0.3284 & 0.3312 \\
-0.01 & 0.3443 & 0.3351 & 0.3304 & 0.3281 & 0.3237 & 0.3263 \\
-0.1 & 0.3310 & 0.3139 & 0.2880 & 0.2749 & 0.2743 & 0.2753 \\
-1 & 0.2670 & 0.2474 & 0.2326 & 0.2290 & 0.2315 & 0.2314 \\
-10 & 0.2409 & 0.2277 & 0.2225 & 0.2219 & 0.2249 & 0.2246 \\
-100 & 0.2376 & 0.2254 & 0.2215 & 0.2212 & 0.2242 & 0.2241 \\
\hline \hline
\end{tabular}




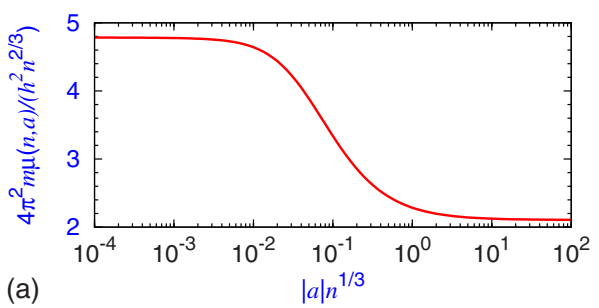

(a)

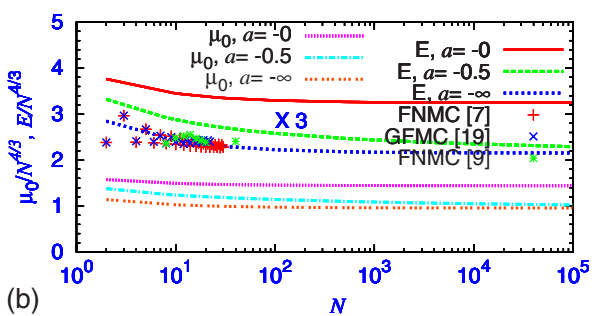

FIG. 2. (Color online) (a) Dimensionless bulk chemical potential $4 \pi^{2} m \mu(n, a) /\left(h^{2} n^{2 / 3}\right)$ of a uniform Fermi gas vs $|a| n^{1 / 3}$ for $a<0$. (b) Chemical potential and energy $\mu_{0}(N, a) / N^{4 / 3}$ and $E(N, a) / N^{4 / 3}$ of a trapped Fermi gas vs $N$ for $a=-0,-0.5,-\infty$. The energies of FNMC [7,9] and GFMC [20] calculations at unitarity are also shown. (Energies and scattering lengths are expressed in oscillator units.)

universal curves yield the simple formula for the energy of a large system with $N$ atoms in terms of that of a small system with $N^{\prime}$ atoms $\left(N \gg N^{\prime}\right)$,

$$
E(N, \alpha)=N^{4 / 3} \frac{E\left(N^{\prime}, \alpha\right) / N^{\prime 2 / 3}-\gamma}{N^{\prime 2 / 3}},
$$

with $\gamma=0.37$, and where we have neglected the small constant 0.37 compared to the large quantity $E(N, \alpha) / N^{2 / 3}$. When $N$ and $N^{\prime}$ are both large, formula (8) becomes $E(N, \alpha)=E\left(N^{\prime}, \alpha\right)\left(N / N^{\prime}\right)^{4 / 3}$. We note that, in Eq. (8), energies are to be considered for the same $\alpha$ and not $a$. We indeed calculated the energies for $N=10^{5}$ atoms using the data for $N^{\prime}=4$ and 10 . The predictions so obtained for $N^{\prime}$ $=4,10$, listed Table I, compare well with the calculated re-
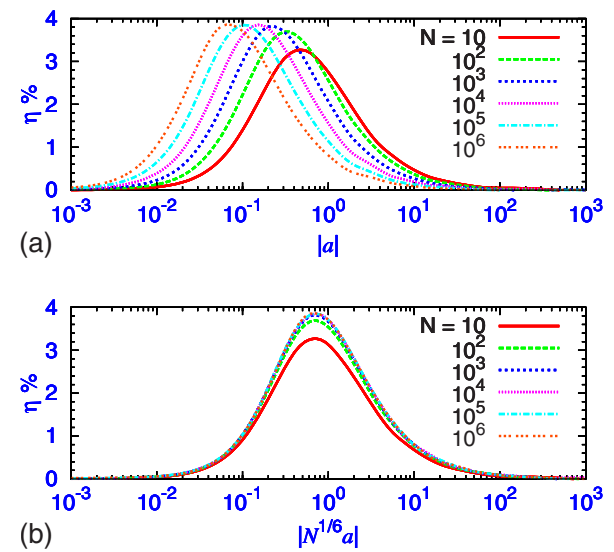

FIG. 3. (Color online) (a) The plot of $\eta=100[E(N, a)$ $\left.-2\left\langle E_{\mathrm{pot}}\right\rangle\right] / E(N, a)$ for different $N$ vs $|a|$; and (b) $\eta=100[E(N, \alpha)$ $\left.-2\left\langle E_{\mathrm{pot}}\right\rangle\right] / E(N, \alpha)$ vs $|\alpha|, \alpha \equiv N^{1 / 6} a$. Scattering lengths are expressed in oscillator units.
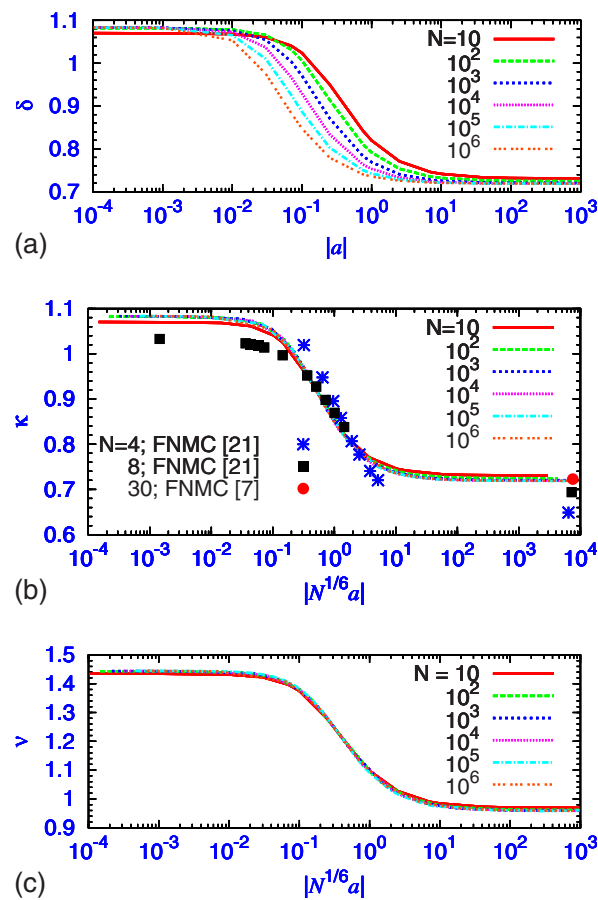

FIG. 4. (Color online) (a) $\delta \equiv\left[E(N, a) / N^{2 / 3}-0.37\right] / N^{2 / 3}$ vs $|a|$ for different $N$ for a trapped Fermi gas from Eq. (1). (b) $\kappa \equiv\left[E(N, \alpha) / N^{2 / 3}-0.37\right] / N^{2 / 3}$ vs $|\alpha|$ for different $N$ from Eq. (1) and FNMC method [21]. (c) $\nu \equiv\left[\mu_{0}(N, \alpha) / N^{2 / 3}-0.27\right] / N^{2 / 3}$ vs $|\alpha|$ for different $N$. (Energies and scattering lengths are expressed in oscillator units.)

sults within about $3 \%$ and $2 \%$ errors, respectively. In Fig. 4(c) we plotted scaled chemical potentials vs $|\alpha|$ for $N=10$ to $10^{6}$ and find that they all lie on the same universal curve. In this case also a prediction of chemical potential for large $N$ using the same for a small $N^{\prime}$ can be made through the scaling formula (8) but now with $\gamma=0.27$ as obtained from an analysis of Fig. 2(b). The predicted $\mu_{0}$ for $N=10^{5}$ using the data for $N^{\prime}=4$ and 10 , listed in Table II, compare well with the calculated results within an error of less than $1.5 \%$.

Because of the universal behavior due to the dominance of Pauli repulsion, other observables of a Fermi superfluid are also correlated with energy and atom number. For example, the root-mean-square radius $\langle r\rangle$ has the scaling $\langle r\rangle$ $\sim N^{1 / 6}$ in BCS and unitarity limits. In Fig. 5 we plot $\langle r\rangle / N^{1 / 6}$ vs $N$ for different $a$. Again there is a slight violation of this scaling for small $N$ and in the crossover region, which could be remedied by a fine-tuning of the type (8). Nevertheless,

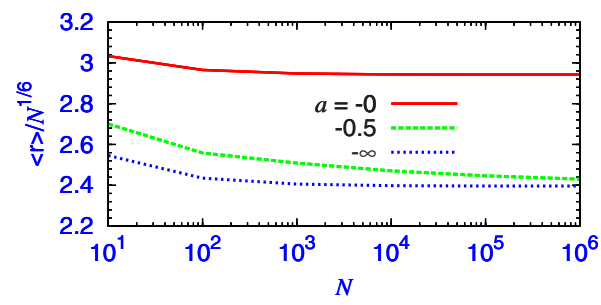

FIG. 5. (Color online) $\langle r\rangle / N^{1 / 6}$ of a trapped Fermi superfluid vs $N$ for different $a=-0,-0.5,-\infty$. (Lengths are expressed in oscillator units.) 
this clearly shows that the radius of a large trapped Fermi superfluid is predictable from the knowledge of radius of a small system not only in the BCS and unitarity limits but also along the crossover. In the case of nuclei, where Pauli repulsion plays an important role, correlations exist among binding energy, radius, and the number of nucleons [26].

\section{CONCLUSION}

In conclusion, from an extensive numerical study of the static properties of a trapped two-component Fermi superfluid using a Galilei-invariant DF formulation [17], equivalent to a generalized hydrodynamic formulation with the correct phase-velocity relation [6], we establish that, because of the dominance of the Pauli repulsion, the trapped Fermi superfluid has a universal behavior not only in the BCS and unitarity limits but also in the crossover region. This allows for a prediction of the static properties (energy, chemical potential, rms radius, etc.) of a large Fermi superfluid in the crossover region from a knowledge of the same of a small system through a scaling relation, cf. Eq. (8). The thus predicted energy and chemical potential of a system with $10^{5}$ atoms from a knowledge of the same with 4 (10) atoms is found to have an error of less than $3 \%$ (2\%). Actually, for small systems one needs to introduce finite-size effects, such as, higher-order gradient corrections and shell effects. The 2-3\% discrepancy quantifies the contribution of such effects. Although we used a DF formulation in the present study, due to the dominance of Pauli repulsion and existence of robust scalings, we do not believe our conclusion to be so peculiar as to have no general validity.

\section{ACKNOWLEDGMENTS}

FAPESP, CNPq (Brazil), and the Institute for Mathematical Sciences of National University of Singapore (IMSNUS) provided partial support. Research was (partially) completed while S.K.A. was visiting IMSNUS.
[1] R. D. Amado, Annu. Rev. Nucl. Sci. 19, 61 (1969).

[2] L. Tomio, A. Delfino, and S. K. Adhikari, Phys. Rev. C 35, 441 (1987).

[3] S. K. Adhikari and A. Ghosh, Phys. Rev. B 55, 1110 (1997).

[4] G. A. Baker, Jr., Phys. Rev. C 60, 054311 (1999).

[5] S. Y. Chang, V. R. Pandharipande, J. Carlson, and K. E. Schmidt, Phys. Rev. A 70, 043602 (2004); J. Carlson, S.-Y. Chang, V. R. Pandharipande, and K. E. Schmidt, Phys. Rev. Lett. 91, 050401 (2003).

[6] S. Giorgini et al., Rev. Mod. Phys. 80, 1215 (2008).

[7] D. Blume, J. von Stecher, and C. H. Greene, Phys. Rev. Lett. 99, 233201 (2007).

[8] J. von Stecher, C. H. Greene, and D. Blume, Phys. Rev. A 77, 043619 (2008).

[9] D. Blume, Phys. Rev. A 78, 013635 (2008).

[10] F. Werner and Y. Castin, Phys. Rev. Lett. 97, 150401 (2006); A. Bulgac and G. F. Bertsch, ibid. 94, 070401 (2005).

[11] E. Braaten et al., Phys. Rep. 428, 259 (2006); H. Heiselberg, Phys. Rev. A 63, 043606 (2001); S. K. Adhikari and A. Ghosh, J. Phys. A 30, 6553 (1997).

[12] D. M. Eagles, Phys. Rev. 186, 456 (1969); A. J. Leggett, J. Phys. (Paris), Colloq. 41, C7-19 (1980); S. K. Adhikari, M. Casas, A. Puente, A. Rigo, M. Fortes, M. A. Solis, M. de Llano, A. A. Valladares, and O. Rojo, Phys. Rev. B 62, 8671 (2000); P. Nozières and S. Schmitt-Rink, J. Low Temp. Phys. 59, 195 (1985).

[13] G. E. Astrakharchik, J. Boronat, J. Casulleras, and S. Giorgini, Phys. Rev. Lett. 93, 200404 (2004); 95, 230405 (2005).

[14] M. Greiner et al., Nature (London) 426, 537 (2003); T. Bourdel, L. Khaykovich, J. Cubizolles, J. Zhang, F. Chevy, M. Teichmann, L. Tarruell, S. J. J. M. F. Kokkelmans, and C. Salomon, Phys. Rev. Lett. 93, 050401 (2004); C. Chin et al.,
Science 305, 1128 (2004); J. Kinast et al., Phys. Rev. Lett. 92, 150402 (2004); K. O'Hara et al., Science 298, 2179 (2002).

[15] M. Bartenstein, A. Altmeyer, S. Riedl, S. Jochim, C. Chin, J. H. Denschlag, and R. Grimm, Phys. Rev. Lett. 92, 203201 (2004).

[16] L. N. Oliveira, E. K. U. Gross, and W. Kohn, Phys. Rev. Lett. 60, 2430 (1988).

[17] S. K. Adhikari and L. Salasnich, Phys. Rev. A 78, 043616 (2008); New J. Phys. 11, 023011 (2009).

[18] Y. E. Kim and A. L. Zubarev, Phys. Rev. A 70, 033612 (2004); N. Manini and L. Salasnich, ibid. 71, 033625 (2005).

[19] J. Kinast et al., Science 307, 1296 (2005); T. Bourdel, J. Cubizolles, L. Khaykovich, K. M. F. Magalhães, S. J. J. M. F. Kokkelmans, G. V. Shlyapnikov, and C. Salomon, Phys. Rev. Lett. 91, 020402 (2003); M. Bartenstein, A. Altmeyer, S. Riedl, S. Jochim, C. Chin, J. H. Denschlag, and R. Grimm, ibid. 92, 120401 (2004); J. T. Stewart, J. P. Gaebler, C. A. Regal, and D. S. Jin, ibid. 97, 220406 (2006); G. B. Partridge et al., Science 311, 503 (2006).

[20] S. Y. Chang and G. F. Bertsch, Phys. Rev. A 76, 021603(R) (2007).

[21] J. von Stecher, C. H. Greene, and D. Blume, Phys. Rev. A 76, 053613 (2007); D. Blume (private communication), kindly provided the data for energy of fixed node Monte Carlo calculation of four and eight trapped fermions reported in Fig. 4(b).

[22] T. D. Lee et al., Phys. Rev. 105, 1119 (1957).

[23] S. K. Adhikari, Phys. Rev. A 77, 045602 (2008).

[24] A. Bulgac, Phys. Rev. A 76, 040502(R) (2007).

[25] J. E. Thomas, J. Kinast, and A. Turlapov, Phys. Rev. Lett. 95, 120402 (2005).

[26] J. M. Blatt and V. F. Weisskopf, Theoretical Nuclear Physics (Wiley, New York, 1952). 